\title{
Viable Real-Time PCR in Environmental Samples: Can All Data Be Interpreted Directly?
}

\author{
Mariana Fittipaldi • Francesc Codony • \\ Barbara Adrados • Anne K Camper • Jordi Morató
}

Received: 19 June 2010 / Accepted: 25 June 2010 /Published online: 15 July 2010

(C) Springer Science+Business Media, LLC 2010

\begin{abstract}
Selective nucleic acid intercalating dyes - ethidium monoazide (EMA) and propidium monoazide (PMA)represent one of the most successful recent approaches to detect viable cells (as defined by an intact cell membrane) by PCR and have been effectively evaluated in different microorganisms. However, some practical limitations were found, especially in environmental samples. The aim of this work was to show that in the application of viable real-time PCR, there may be significant biases and to propose a strategy for overcoming some of these problems. We present an approach based on the combination of three real-time PCR amplifications for each sample that should provide an improved estimation of the number of viable cells. This approach could be useful especially when it is difficult to determine a priori how to optimize methods using PMA or EMA. Although further studies are required to improve viable real-time PCR methods, the concept as outlined here presents an interesting future research direction.
\end{abstract}

M. Fittipaldi $\cdot$ F. Codony $(\bowtie) \cdot$ B. Adrados $\cdot$ J. Morató Laboratori de Microbiologia Sanitària i Mediambiental (MSMLab)-Aquasost, UNESCO Chair in Sustainability, Universitat Politècnica de Catalunya,

C/Violinista Vellsolà 37,

08222 Terrassa, Barcelona, Spain

e-mail: codony@oo.upc.edu

M. Fittipaldi · A. K Camper

Center for Biofilm Engineering, Montana State University,

366 EPS Building,

Bozeman, MT, USA

\section{Introduction}

PCR is a rapid and sensitive technique for microbial detection. However, until a few years ago, it was assumed that a major disadvantage of this method was its inability to differentiate between viable and nonviable cells [27] and the overestimation of potentially viable biomass. After the first publication of a real-time PCR procedure using ethidium monoazide (EMA), this premise began to change [17].

The utilization of selective nucleic acid intercalating dyes, like EMA and propidium monoazide (PMA), has been suggested as a means to reduce PCR signals from DNA originating from dead cells $[5,12,17,22]$. Therefore, it is one of the most successful approaches to detect viable cells by PCR (herein called v-PCR). The approach is based on membrane integrity to distinguish between viable and nonviable cells. Theoretically, selective nucleic acid intercalating dyes should only penetrate into membranecompromised cells or dead cells. The presence of an azide group is believed to permit cross-linking of the dye to the DNA after exposure to strong visible light. The photolysis of EMA and PMA converts the azide group into a highly reactive nitrene radical, which can react with any organic molecule in its proximity, including the bound DNA. In this bound state, the DNA cannot be amplified by PCR [13, 22]. At the same time when the cross-linking with DNA occurs, the light reacts unbound excess dye with water molecules. The resulting hydroxylamine is no longer reactive, so the DNA from cells with intact membranes is supposedly not modified in the DNA extraction procedure [16].

The use of EMA or PMA has been effectively evaluated in different bacteria $[2,5,7,16,18,22,23]$, spores [21], fungi [25], and virus [9]. However, there is evidence demonstrating that v-PCR using DNA-intercalating dyes 
has practical and theoretical limitations [15, 19, 24, 26]. In some cases, most drawbacks may be reduced considerably by the development of precise procedures adapted to each sample or microorganism, and researchers are considering the use of this approach in their present and future work. It is clear that with the use of these techniques, our vision of microbial dynamics in most of areas of microbiology, including environmental and clinical microbiology and quality control, will be more exact or at least quite different.

Despite this exciting perspective, to improve the application of these dyes for complex environmental use, a critical discussion about the need to establish common interpretation of $\mathrm{v}-\mathrm{PCR}$ in environmental samples is warranted. The ideal situation ( 1 in Fig 1 ) is when all DNA present in the sample may be detected with real-time PCR and all DNA from viable microorganisms may be detected by v-PCR. As expected, several critical points need to be considered ( 2 in Fig 1). Firstly, the dye should not penetrate live cells. However, some studies have demonstrated that EMA may penetrate cells with intact membranes $[5,8,10,12,14]$, with the extent of EMA uptake by intact cells dependent on the bacterial species [14] and the EMA concentration [28]. PMA has been proposed as a more appropriate alternative due to a comparative study showing that PMA is efficiently excluded from cells with intact cell membranes [14]. For that reason, PMA was used in this work. It is also probable that DNA-intercalating dyes will have access to the DNA in viable cells with reversibly damaged membranes; these cells are likely to be present in environmental samples. In both of these cases, cross-linkage will produce false negative results. This is fraction $a$ in 2 and 3 in Fig 1. Secondly, dye or light may not be able to penetrate all dead microorganisms and overestimate the number of viable cells (false positives). This is fraction $b$ in 2 and 3 in Fig 1. An example of a condition leading to false positives would be the presence of high levels of suspended solids or biomass in water samples that could inhibit the cross-linking step by light activation since the radiation probably will not be able to penetrate through the liquid [24, 26]. Likewise, for central cells within clusters, biofilms [19], cells embedded in encrustations or precipitates, nucleic DNA from eukaryotes, cysts or other resistant forms, and cells inside protozoa (symbiotic or parasites), the penetration of dye into cells might be limited or not occur. To overcome that, in some cases as with bacterial spores, longer incubation times have been proposed [21]. Furthermore, the dye could undergo chemical adsorption onto different compounds present in the sample. Another specific example is that EMA shows a variable yield of the photo-cross-linking reaction at different salt concentrations [4]. Subsequently, it is likely that halophiles will need customized procedures to maximize dye performance and minimize cell death during treatment as a consequence of osmotic alterations. The presence of substantial amounts of dead cells also could interfere with DNA extraction or amplification from viable cells. For example, enumeration of viable Listeria monocytogenes and Escherichia coli O157:H7 cells by v-PCR was impacted, although not strongly, by the presence of dead cells $[18,28]$. The effect of this last factor may be experimentally minimized by means of extensive optimization procedures, including increasing dye concentration and incubation time.

As shown in 3 in Fig. 1, the current v-PCR method will detect the maximum number of viable cells, which includes the minimum number of viable cells $(\mathrm{mV})$ and false
Figure 1 Different theoretical possibilities when photoactivable intercalating dyes are used for v-PCR. 1 Theoretical approach: $\mathrm{v}$-PCR detects all viable cells. 2 Viable PCR method has limitations. Fraction $a$ : the dye may be able to penetrate into viable or reversibly damaged cells; fraction $b$ : not all DNA from dead cells is inactivated by pretreatment with the dye. 3 Approach for the estimation of the minimum number of viable cells $(\mathrm{mV})$ using a combination of three PCR assays for each sample
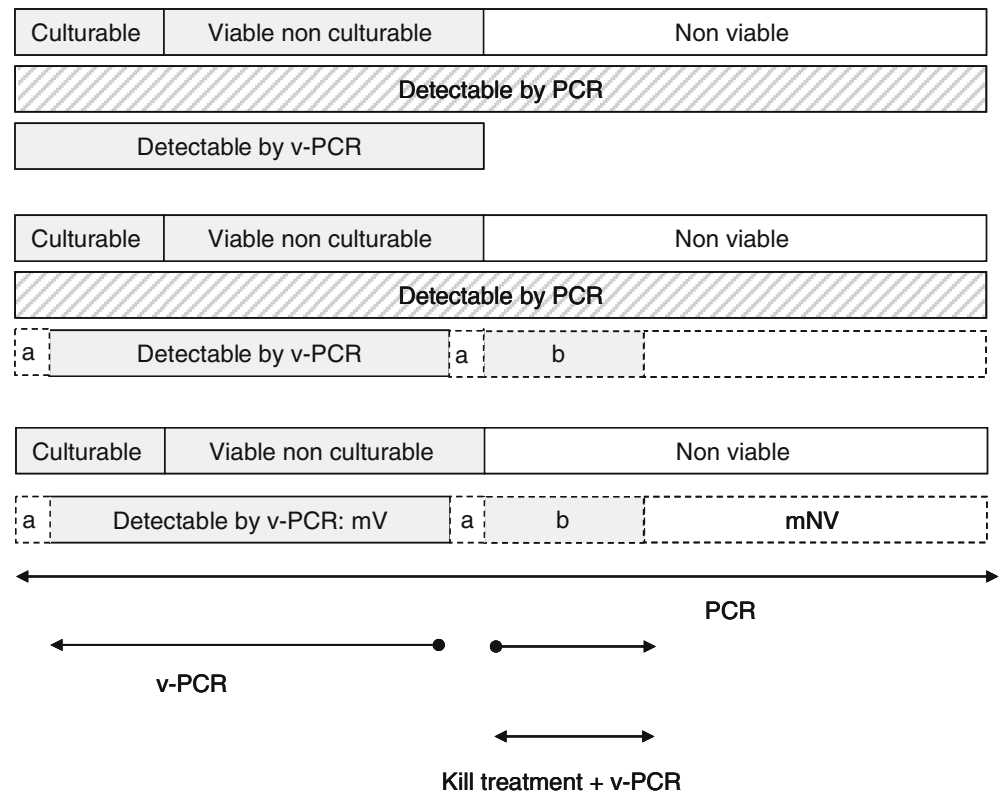
positive live cells (fraction $b$ ). Traditional PCR detects all organisms, including viable and nonviable cells. The difference between the two methods gives the maximum number of nonviable cells which includes the minimum number of nonviable cells (mNV) and false negative dead cells (fraction $a$ ). As shown in 3 in Fig. 1, an improvement of the detection of the viable cell fraction can be attained using an additional PCR approach. First, a rough estimation of fraction $a$ for a specific organism can be obtained using real-time PCR, v-PCR, and pure cultures in exponential growth and assuming that this situation represents the optimal physiological condition for intact membranes. The real-time PCR results would give the minimum number of true negative cells, and the difference between real-time PCR and v-PCR results would give an estimation of the fraction of false negatives (fraction $a$ ) that should be negligible. The values for fraction $b$ can be estimated by combining a v-PCR test with an additional assay that consists of killing all cells and combining with a subsequent $\mathrm{v}$-PCR test. The value of $\mathrm{v}-\mathrm{PCR}$ in the original sample less the value of "kill treatment + v-PCR" (herein called k\&v-PCR), assuming that all cells are killed, would give an estimation of the fraction of false positives (fraction $b$ ). Considering the estimate of fraction $a$ and fraction $b$, the minimum viable cell number can be calculated. This number will be a more accurate representation of the number of organisms in a sample that may pose a public health concern or act as an indicator of the quality of a product or the efficacy of a disinfection technology. By also using a PCR assay without PMA, the difference between PCR and v-PCR along with the estimation of fraction $a$ will let us more closely estimate the minimum number of nonviable cells.

The objective of our work was to show that there exist many factors that should be considered in the application of $\mathrm{v}$-PCR and to stimulate research and discussion regarding these issues. We present preliminary data outlining the approach explained above that it is based on the combination of three real-time PCR amplifications for each sample, which can improve viable cell number estimation using nucleic acid amplification methods. At a minimum, these results will lead to a better understanding and a more realistic interpretation of the number of viable cells in a sample.

\section{Material and Methods}

The three-pronged PCR approach was used to determine the viability of Legionella pneumophila in artificially inoculated treated secondary wastewater effluent after disinfection.

L. pneumophila serogroup 1 (NCTC12821) was cultured in GVPC agar (OXOID, UK). Once the culture was in exponential growth phase (3-day culture), a bacterial suspension was prepared by transferring single colonies into a tube with sterile saline solution and adjusting the $\mathrm{OD}_{600}$ to 0.2 , which approximately corresponds to a concentration of $10^{8}$ cells $\mathrm{mL}^{-1}$, as confirmed by plate count. A decimal dilution of the bacterial suspension was inoculated in $250 \mathrm{~L}$ of wastewater effluent to obtain an approximate concentration of $10^{2}$ cells $\mathrm{mL}^{-1}$. The treated secondary wastewater was held in a $20-\mathrm{m}^{3}$ reservoir tank that fed two different disinfection systems: chlorination and an advanced oxidation technology (AOT) that utilizes ozone, photolysis, and photocatalytic decomposition by ultraviolet light. Each disinfection system consisted of a 250-L tank where the L. pneumophila inoculation took place, a recirculation loop and a disinfection step-a chlorinator for chlorination and a Benrad water purification facility (Benrad Co., Sweden) for the AOT system. The Benrad facility has a simple structure consisting of a titanium pipe with a titanium oxide layer on the inner wall and an ultraviolet lamp placed in the center. Both disinfection systems work automatically by means of a programmable controller. Different treatments can be applied by changing chlorine concentration and recirculation times for chlorination or AOT. For these experiments, three disinfection treatments were evaluated: $3 \mathrm{ppm}$ of chlorine and $30 \mathrm{~min}$ of recirculation, $3 \mathrm{ppm}$ of chlorine and $60 \mathrm{~min}$ of recirculation, and AOT with a recirculation time of $60 \mathrm{~min}$. Water samples of 11 were collected in sterilized bottles with sodium thiosulfate $(1 \mathrm{~mL} / \mathrm{L}, 3 \%)$.

Each sample of $300 \mathrm{~mL}$ was concentrated by membrane filtration using a nylon membrane $(0.45-\mu \mathrm{m}$ pore diameter, Millipore). Cells were resuspended in $7 \mathrm{~mL}$ of saline solution by vigorous vortexing for $60 \mathrm{~s}$ with five glass beads (5-mm diameter) and sonication for $3 \mathrm{~min}$ in an ultrasound water bath- $40-\mathrm{W}$ power, $40-\mathrm{kHz}$ ultrasound frequency (JP Selecta, Barcelona, Spain). The cell suspension was split into three aliquots of $2 \mathrm{~mL}$ each. They were concentrated by centrifugation $(14,000 \times g$ for $5 \mathrm{~min})$ using a minicentrifuge (Minispin Plus-Eppendorf, Hamburg, Germany) and discarding the supernatant to obtain a pellet.

One of the aliquots was treated with PMA for the v-PCR assay. Briefly, PMA (Biotium, Inc., Hayward, California) was dissolved in $20 \%$ dimethyl sulfoxide (Sigma) to create a stock concentration of $2 \mathrm{mM}$ and stored at $-20^{\circ} \mathrm{C}$ in the dark. The bacterial pellet was resuspended with $190 \mu \mathrm{L}$ of $1 \times$ phosphate-buffered saline (PBS), $\mathrm{pH} 7.4$, in a propylene $1.5-\mathrm{mL}$ minicentrifuge tube, and $10 \mu \mathrm{L}$ of $2 \mathrm{mM}$ PMA stock solution was rapidly added in a darkened room. The resultant cell suspension was incubated for $5 \mathrm{~min}$ in the dark at room temperature to allow PMA to enter into the cells with compromised or damaged membranes. The samples were then photoactivated for $15 \mathrm{~min}$ using Led-Active Blue photoactivation system (IB-Applied Science, Barcelona, 
Spain). After photo-induction of cross-linking, cells were pelleted by centrifugation at $14,000 \times g$ for $5 \mathrm{~min}$. The supernatant was discarded and the pellet was resuspended in $200 \mu \mathrm{L}$ of PBS. The second aliquot was exposed to isopropanol (final concentration, $70 \%$ ) for $15 \mathrm{~min}$. Isopropanol treatment induces membrane damage; the assumption that isopropanol kills the cells not inactivated by disinfection was made since this is the same approach used in the development of the v-PCR method [14]. Isopropanol was removed by harvesting cells by centrifugation at $14,000 \times g$ for $5 \mathrm{~min}$ prior to resuspension in $200 \mu \mathrm{L}$ of PBS. After that, the bacterial suspension was treated with PMA following the protocol described above and was used for the k\&v-PCR assay. Loss of culturability of isopropanol-treated cells was verified by streaking $200 \mu \mathrm{L}$ of cell suspension and dilutions on GVPC agar plates (Oxoid) followed by incubation at $37^{\circ} \mathrm{C}$ for 10 days The third aliquot also was resuspended in $200 \mu \mathrm{L}$ PBS and was used for the PCR assay.

In all cases, DNA was extracted with EZNA tissue DNA purification kit (Omega Bio-Tek, Norcross, GA, USA) according to the manufacturer's instructions.

Real-time PCR analysis was performed on a Ligthcycler 1.5 (Roche Molecular Diagnostic, Manheim, Germany). The reaction mixture was composed of $10 \mu \mathrm{L}$ of Fast Start Taqman Probe Master (Roche Molecular Diagnostic), $0.4 \mathrm{U}$ of Uracil-DNA-glycosylase (New England BioLabs, Ipswich, MA, USA), $9 \mu \mathrm{L}$ of sample, and $0.45 \mu \mathrm{M}$ of $L$. pneumophila-specific mip primers LPQF 5'-TTCATTTGYTGYTCGGTTAAAGC and LPQR 5'-AWTGGCTAAAGGCATGCAAGAC which delimited a 66-bp DNA fragment [3], and $0.1 \mu \mathrm{M}$ of mip-specific Taqman hybridization probe (5'-AGCGCCACTCATAG) labeled at the $5^{\prime}$ end with a FAM reporter dye and at the $3^{\prime}$ end, with a non-fluorescent quencher and conjugated to a MGB [3]. The experimental protocol consisted of one step of $2 \mathrm{~min}$ at $50^{\circ} \mathrm{C}$ to allow UDG to break down the possible contaminating amplicons, one step of $15 \mathrm{~min}$ at $95^{\circ} \mathrm{C}$ for Taq polymerase activation, and 45 cycles $\left(95^{\circ} \mathrm{C}\right.$ for $15 \mathrm{~s}, 60^{\circ} \mathrm{C}$ for $60 \mathrm{~s}$ ) for DNA amplification.

The DNA used as standard reference for L. pneumophila quantification was prepared according to AFNOR XP T90471 [1]. L. pneumophila serogroup 1 (NCTC12821) was used as a reference strain. A standard DNA curve was established using a 3-day culture in GVPC agar (OXOID). Once the culture was ready, a bacterial suspension was prepared by transferring single colonies into a tube with sterile saline solution and adjusting the $\mathrm{OD}_{600}$ to 0.2 , which approximately corresponds to a concentration of $10^{8}$ cells $\mathrm{mL}^{-1}$, as confirmed by plate count. Serial tenfold dilutions were prepared from the bacterial suspension using sterile saline solution to obtain the set of dilutions that was later used for the standard curve. DNA also was obtained with the EZNA tissue DNA purification kit (Omega Bio-Tek) according to the manufacturer's instructions. Amplification efficiency $(100 \%)$ was estimated by means of the slope calculation method from a calibration dilution curve [20].

For each assay, the threshold cycle $(\mathrm{Ct})$ was determined to quantify each DNA product. Quantification was performed including two external standards containing $4.5 \times$ $10^{4}$ cells/L in each set of PCR experiments. The cell number of each sample was determined by comparison to each standard. Each sample was tested in duplicate and mean values were calculated. A negative control (PCRgrade water, QIAGEN, Valencia, CA, USA) was included in all assays.

Statistical analyses to calculate mean values and the standard error were performed using Microsoft Excel.

\section{Results and Discussion}

Recent modifications of the PCR technique involving pretreatment of samples with EMA or PMA prior to DNA extraction have been reported to differentiate viable and dead microbial cells $[2,7,12,14-16,22]$. Although membrane integrity is an incomplete criterion for cell viability, the v-PCR approach has received positive evaluation in several publications. The v-PCR approach is viewed as an important step toward the ability to study live cells using PCR-based methods [13]. In particular, the v-PCR approach has the potential to substantially improve the data on waterborne exposures to several microorganisms on disinfection efficacy evaluations and enhance the validity of human risk assessment by DNA-based detection methods. A fundamental drawback, however, is that the principle is based on membrane integrity as a viability criterion [13]. The method does not allow for monitoring the killing efficacy by other inactivation mechanisms that do not directly target the cell membrane, like ultraviolet light irradiation [13], and it will produce biases in samples that contain viable cells with reversibly damaged membranes. These issues illustrate the need for critical discussions so that the method can be further improved.

In the present study, the v-PCR approach resulted in a positive DNA amplification signal when it was used with theoretically dead cells. This outcome suggests that v-PCR yields false positive results. Similar results have been observed with other bacteria in previous reports $[2,6,11]$. We noted that the presence of a high number of dead cells is one possible reason for false positive detection signals, and other researchers have reached similar conclusions [28]. Optimization of the PMA method, by modifying variables such as the concentration of PMA, incubation time, light source, distance from light source, and light exposure time, can help reduce false-positive results, but as illustrated in the 
"Introduction," there may be many factors that could cause significant biases in the application of v-PCR.

The above named limitations in the current v-PCR method led us to seek methods to better estimate the number of viable cells, excluding false positive signals, to improve the ability to assess survival of target organisms exposed to environmental stress and disinfection. The modified v-PCR approach was used to detect $L$. pneumophila suspended in a complex environmental water (treated secondary wastewater effluent) disinfected with chlorine and an advanced oxidation protocol. Wastewater without disinfection treatment was used as a control. L. pneumophila were detected by real-time PCR. Samples included: (1) realtime PCR without PMA pretreatment to give an estimate of the total L. pneumophila population in wastewater samples with and without disinfection; (2) real-time PCR with PMA pretreatment to give an estimate of the viable population in the disinfected samples and wastewater without disinfection sample; and (3) real-time PCR with PMA pretreatment after isopropanol treatment of the wastewater samples with and without disinfection to inactive the remaining organisms and to obtain an estimate of the minimum viable cell number.

Regarding the variability among PCR estimates of "the total population," we found that the coefficient of variation was $0.95 \%$. The obtained results show that for the type of analyzed samples and for our target organism, a fraction of viable cells may be due to false positive amplification (Fig. 2). Moreover, we observed that the fraction of false positives increased with the number of dead cells present in the sample. For example, after $30 \mathrm{~min}$ of treatment with chlorine, about $1.9 \%$ of the total number of L. pneumophila cells were viable and about $40 \%$ of these viable cells may be false positive if only a direct v-PCR was performed. In the case of $60 \mathrm{~min}$ of treatment with chlorine, the percentage of false positives in the viable cells number was about $18 \%$. However, after $60 \mathrm{~min}$ of treatment with photocatalysis, about $12.5 \%$ of cells were viable and about $7.5 \%$ of viable cells may be false positives if only a direct v-PCR was performed. Consequently, the data obtained using the additional step of isopropanol treatment were encouraging and indicate that the approach presented in this work is reasonable.

Our results also emphasize the need for focusing on the sources of false positives and false negatives in future research. Understanding how these sources of error can be experimentally mitigated is ultimately necessary to obtain a better understanding of the sample complexity.

Addressing these errors will ensure wider acceptance of nucleic acid-based methods for assessing the viability of target pathogens in environmental samples. A realistic approach for assessing the minimum number of viable cells in undefined environmental samples was created based on three real-time PCR assays. This approach was tested and the bias due to false
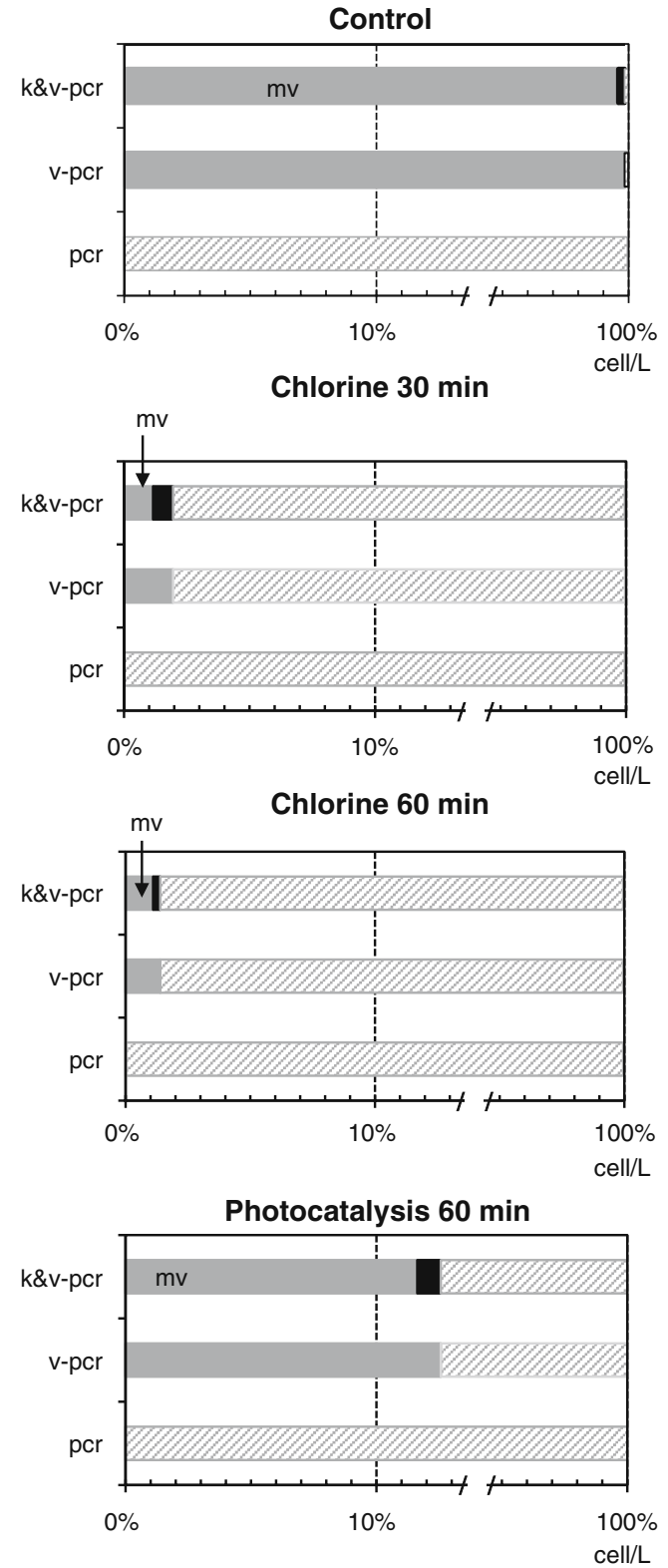

Figure 2 Presence of $L$. pneumophila in disinfected wastewater samples measured with three approaches: 1 Real-time PCR ( $p c r) ; 2$ PMA treatment and real-time PCR (v-pcr); 3 Killing treatment (with isopropanol), PMA treatment, and real-time PCR ( $k \& v-p c r)$. Gray bars show the results of Legionella viable levels after each treatment. Black bars show the false positive Legionella levels after k\&v-PCR. Hatched gray bars represent total Legionella number cells for the PCR approach and the dead cells number for both, v-pcr and k\&v-pcr, approaches. The $\mathrm{mV}$ level indicates the minimum viable Legionella fraction. Results were obtained from replicate samples

positives has been estimated. At least for these samples, this particular microbial target, and cell damage caused by disinfection, the feasibility of the approach for assessing the minimum number of viable cells with PMA has been validated. In the absence of robust and reliable procedures, the concept as outlined here is interesting as a future research 
direction and also offers a better understanding of microbial dynamics in complex samples.

Although the PMA approach is an important step forward in the quantification of viable cells by DNA detection-based methods, there are many factors that may lead to erroneous results when this approach is applied in environmental samples. Because in microbiology it is very difficult to have accurate results, we believe that the outlined approach of determining the minimum number of viable cells is a step forward.

Acknowledgments We are most grateful to the Comissionat per a Universitats $i$ Recerca del Departament d'Innovació, Universitats $i$ Empresa de la Generalitat de Catalunya i del Fons Social Europeu for the financial support received from the program of pre-doctoral scholarships FI and the program of Beques per a estades de recerca fora de Catalunya (BE 2008). This work was supported by Agència Catalana de Seguretat Alimentària and by the Ministerio de Ciencia e Innovación with grant CTM2008-06676-C05-02/TECNO to Jordi Morató.

\section{References}

1. AFNOR XP T90-471 (2006) Détection et quantification des Legionella et/ou Legionella pneumophila par concentration et amplification génique par reaction de polymérisation en chaîne (PCR). Normalization Française

2. Bae S, Wuertz S (2009) Discrimination of viable and dead fecal bacteroidales bacteria by quantitative PCR with propidium monoazide. Appl Environ Microbiol 75:2940-2944

3. Behets J, Declerck P, Delaedt Y, Creemers B, Ollevier F (2007) Development and evaluation of a Taqman duplex real-time PCR quantification method for reliable enumeration of Legionella pneumophila in water samples. J Microbiol Methods 68:137-144

4. Bolton PH, Kearns DR (1978) Spectroscopic properties of ethidium monoazide: a fluorescent photoaffinity label for nucleic acids. Nucleic Acids Res 5:4981-4903

5. Cawthorn DM, Witthuhn RC (2008) Selective PCR detection of viable Enterobacter sakazakii cells utilizing propidium monoazide or ethidium bromide monoazide. J Appl Microbiol 105:11781185

6. Chang B, Taguri T, Sugiyama K, Amemura-Maekawa J, Kura F, Watanabe H (2010) Comparison of ethidium monoazide and propidium monoazide for the selective detection of viable Legionella cells. Jpn J Infect Dis 63:119-123

7. Delgado Viscogliosi P, Solignac L, Delattre JM (2009) Viability PCR, a culture-independent method for rapid and selective quantification of viable Legionella pneumophila cells in environmental water samples. Appl Environ Microbiol 75:3502-3512

8. Flekna G, Stefanic P, Wagner M, Smulders FJ, Mozina SS, Hein I (2007) Insufficient differentiation of live and dead Campylobacter jejuni and Listeria monocytogenes cells by ethidium monoazide (EMA) compromises EMA/real-time PCR. Res Microbiol 158:405-412

9. Graiver DA, Saunders SE, Topliff CL, Kelling CL, Bartelt-Hunt SL (2010) Ethidium monoazide does not inhibit RT-PCR amplification of nonviable avian influenza RNA. J Virol Methods 164:51-54. doi:10.1016/j.jviromet.2009.11.024

10. Kobayashi H, Oethinger M, Tuohy MJ, Hall GS, Bauer TW (2009) Unsuitable distinction between viable and dead Staphylo- coccus aureus and Staphylococcus epidermis by ethidium bromide monoazide. Lett Appl Microbiol 48:633-638

11. Kralik P, Nocker A, Pavlik I (2010) Mycobacterium avium subsp. paratuberculosis viability determination using F57 quantitative PCR in combination with propidium monoazide treatment. Int $\mathrm{J}$ Food Microbiol. doi:10.1016/j.ijfoodmicro.2010.03.018

12. Nocker A, Camper AK (2006) Selective removal of DNA from dead cells of mixed bacterial communities by use of ethidium monoazide. Appl Environ Microbiol 72:1997-2004

13. Nocker A, Camper AK (2009) Novel approaches toward preferential detection of viable cells using nucleic acid amplification techniques. FEMS Microbiol Lett 291:137-142

14. Nocker A, Cheung CY, Camper AK (2006) Comparison of propidium monoazide and ethidium monoazide for differentiation of live vs. dead bacteria by selective removal of DNA from dead cells. J Microbiol Meth 67:310-320

15. Nocker A, Sossa-Fernandez P, Burr MD, Camper AK (2007) Use of propidium monoazide for live/dead distinction in microbial ecology. Appl Environ Microbiol 73:5111-5117

16. Nocker A, Mazza A, Masson L, Camper AK, Brousseau R (2009) Selective detection of live bacteria combining propidium monoazide sample treatment with microarray technology. J Microbiol Methods 76:253-261

17. Nogva HK, Dromtorp SM, Nissen H, Rudi K (2003) Ethidium monoazide for DNA-based differentiation of viable and dead bacteria by $5^{\prime}$-nuclease PCR. Biotechniques $810: 812-813$

18. Pan Y, Breidt F (2007) Enumeration of viable Listeria monocytogenes cells by real-time PCR with propidium monoazide and ethidium monoazide in the presence of dead cells. Appl Environ Microbiol 73:8028-8031

19. Pisz JM, Lawrence JR, Schafer AN, Siciliano SD (2007) Differentiation of genes extracted from nonviable versus viable micro-organisms in environmental samples using ethidium monoazide bromide. J Microbiol Methods 71:312-318

20. Rasmussen R (2001) Quantification on the LightCycler. In: Meuer S, Wittwer C, Nakagawara K (eds) Rapid cycle real-time PCR, methods and applications. Springer, Heidelberg, pp 21-34

21. Rawsthorne H, Dock CN, Jaykus LA (2009) PCR-based method using propidium monoazide to distinguish viable from nonviable Bacillus subtilis spores. Appl Environ Microbiol 75:2936-2939

22. Rudi K, Moen B, Drømtorp SM, Holck L (2005) Use of ethidium monoazide and PCR in combination for quantification of viable and dead cells in complex samples. Appl Environ Microbiol 71:1018-1024

23. Soejima T, Iida K, Qin T, Taniai H, Seki M, Takade A, Yoshida S (2007) Photoactivated ethidium monoazide directly cleaves bacterial DNA and is applied to PCR for discrimination of live and dead bacteria. Microbiol Immunol 51:763-775

24. Varma M, Field R, Stinson M, Rukovets B, Wymer L, Haugland R (2009) Quantitative real-time PCR analysis of total and propidium monoazide-resistant fecal indicator bacteria in wastewater. Water Res 43:4790-4801. doi:10.1016/j.watres.2009.05.03

25. Vesper S, McKinstry C, Hartmann C, Neace M, Yoder S, Vesper A (2008) Quantifying fungal viability in air and water samples using quantitative PCR after treatment with propidium monoazide (PMA). J Microbiol Methods 72:180-184

26. Wagner AO, Malin C, Knapp BA, Illmer P (2008) Removal of free extracellular DNA from environmental samples by ethidium monoazide and propidium monoazide. Appl Environ Microbiol 74:2537-2539

27. Wang S, Levin RE (2006) Discrimination of viable Vibrio vulnificus cells from dead cells in real-time PCR. J Microbiol Methods 64:1-8

28. Wang L, Li Y, Mustapha A (2009) Detection of viable Escherichia coli $\mathrm{O} 157: \mathrm{H7}$ by ethidium monoazide real-time PCR. J Appl Microbiol 107:1719-1728 